\title{
The Effect of a Psycho-Educational Intervention on Quality of Life of Patients with Advanced Lung Cancer Undergoing Chemotherapy
}

\author{
Yanli Zhao $^{1^{*}}$, Ruifang Zhu ${ }^{2}$ \\ ${ }^{1}$ Shanxi Cancer Hospital, Taiyuan, China \\ ${ }^{2}$ The First Hospital of Shanxi Medical University, Taiyuan, China \\ Email: *zhaoyanli7832395@163.com
}

Received 8 July 2015; accepted 6 October 2015; published 9 October 2015

Copyright (C 2015 by authors and Scientific Research Publishing Inc.

This work is licensed under the Creative Commons Attribution International License (CC BY). http://creativecommons.org/licenses/by/4.0/

\section{(c) (i) Open Access}

\section{Abstract}

Objective: Cancer patients undergoing chemotherapy commonly experience depression and anxiety, which, along with adverse effects of chemotherapy, inevitably compromise quality of life (QOL) of the patients. The aim of this study was to investigate the effect of a psycho-educational intervention on the quality of life (QOL) of patients with advanced lung cancer undergoing chemotherapy. Methods: Two hundred in-patients with unresectable advanced lung cancer undergoing chemotherapy in Shanxi Cancer Hospital from January to October 2013 were randomized into the intervention group $(n=100)$ and the control group $(n=100)$. Patients in the intervention group received a purposely designed and nurse-delivered psycho-educational intervention in addition to routine caring and chemotherapy, whereas those in the control group underwent chemotherapy and routine caring only. QOL of patients was assessed according to QOL instrument for lung cancer (QLICP-LU). The impact of the psycho-educational intervention on QOL was analyzed using independent-sample $t$ test and $\chi^{2}$ test. Results: The psychological, physical, and social function, and the overall QOL scores were significantly improved in patients who received the psychoeducational intervention compared with those in the control group. Conclusions: A nurse-delivered psycho-educational intervention is a useful strategy to improve QOL of patients with advanced lung cancer undergoing chemotherapy.

\section{Keywords}

Psychological Intervention, Psycho-Educational Intervention, Lung Cancer, Quality of Life

\footnotetext{
${ }^{*}$ Corresponding author.
}

How to cite this paper: Zhao, Y.L. and Zhu, R.F. (2015) The Effect of a Psycho-Educational Intervention on Quality of Life of Patients with Advanced Lung Cancer Undergoing Chemotherapy. Open Journal of Nursing, 5, 843-847. 


\section{Introduction}

Lung cancer is a leading cause of cancer-related mortality in men and women all over the world. In particular, the incidence of lung cancer is rapidly increasing in China with the crude incidence rate being 46.08 per 100,000 in 2010 [1]. In the same year, 605,900 patients were diagnosed and 486,600 patients died of lung cancer in the country [1]. This is closely related to the rapid increase in aged population, environment pollution, and cigarette smoking [1].

Despite recent advances in molecularly targeted therapy and immunotherapy, conventional chemotherapy remains one of the major therapeutic approaches in the treatment of patients with advanced lung cancer [2]. Nevertheless, these patients commonly experience depression and anxiety, which, along with adverse effects of chemotherapy, inevitably compromise their quality of life (QOL) [3] [4]. Effective interventions to improve the psychological, physical, and social function of patients with advanced lung cancer undergoing chemotherapy are urgently needed.

In this study, we have examined the effect of a purposely designed and nurse-delivered psycho-educational intervention on QOL of patients with advanced lung cancer undergoing chemotherapy. We report here that the psycho-educational intervention significantly improves the psychological, physical, and social function, and overall QOL of the patients, and that it reduces common symptoms associated with chemotherapy. These results suggest that nurses can play an important role in assistance of patients in improving their QOL through delivering psycho-educational interventions.

\section{Materials and Methods}

\subsection{Patients and Inclusion and Exclusion Criteria}

In-patients $(n=200)$ with unresectable advanced lung cancer undergoing chemotherapy in Shanxi Cancer Hospital from January to October 2013 were enrolled in this study according to the following criteria: 1) they met the lung cancer diagnostic criteria of the Chinese Common Malignancy Norms; 2) Advanced lung cancer (Stage IIIB to IV) was diagnosed according to the International Union Against Cancer TNM classification in 1997; 3) they were prescribed for chemotherapy; 4) they had accepted at least primary school education; 5) they did not have any heart diseases, liver diseases, kidney disease, systemic infection, severe anemia, and cachexia, and were not complicated with other chronic diseases at the time of commencement of chemotherapy; 6) they agreed to participate in the study and signed informed consent forms. According to the completely randomized grouping design, the patients were numbered chronologically from 1 to 200. Patients with odd numbers were assigned into the intervention group, whereas those with even numbers were assigned to the control group. There were $70.75 \%$ males and $29.25 \%$ females, which ages ranging from 61 to 70 years old. $70.25 \%$ of the enrolled patients had a smoking history longer than 10 years. Chi-square analysis showed that the differences in the general demographic characteristics were not statistically significant between the intervention and control groups.

\subsection{Tools of Quality of Life Instruments for Cancer Patients: Lung Cancer (QLICP-LU)}

The one-scale method developed by Wan Chong Hua, et al. was used to measure the QOT of cancer patients [5], which includes common modules (the physical function, the psychological function, the social function, common symptoms, and side effects) and a specific module that contains eight entries. The reliability of this scale was above 0.7 and its validity was 0.82 [4]. QOL was assessed once one week for each patient throughout the hospitalized period.

\subsection{Therapies}

Patients in both the control group and the intervention group were treated with conventional chemotherapy. At the same time, patients in the control group received routine caring while those in the intervention group received an additional nurse-delivered psycho-educational intervention.

\subsection{Psycho-Educational Intervention}

A three-stage measure was taken according to general psychological conditions of patients: 1) the establishment of a nurse-patient communication platform to assist patients in relieving tensions and fears (nurses are readily 
available to listen and respond to narrations of patients with a sincere attitude); 2) Assistance of patients in establishing a positive attitude towards the disease (nurses respect patients' beliefs, provide guidance, and meet the requirements of the patients); 3) provision of educational counseling to patients and caregivers (nurses explain the disease and the therapeutic process to patients and their caregivers).

\subsection{Statistics}

EpiData Software was used to establish a database. Statistical analysis was performed using SAS 8.0 and SPSS 13.0 software. Independent-sample $t$ test and $\chi^{2}$ test was used to compare the difference between groups.

\section{Results}

\subsection{The Psycho-Educational Intervention Improves QOL of Patients with Advanced Lung Cancer Undergoing Chemotherapy}

We used the one-scale method to measure QOT of cancer patients before and after they recieved the psychoeducational intervention [4]. The results showed that the physical function, the psychological function, and the social function were all significantly improved by the intervention (Table 1). Similarly, the scores for overall QOL were also increased (Table 1). In addition, common symptoms and adverse effects associated with chemotherapy that were included in the specific module domain such as nausea, vomiting, and fatigue were markedly reduced after the intervention (Table 1). Of note, scores for cough was also reduced, although whether this was due to the psycho-educational intervention remains to be determined.

\subsection{QOL of Patients in the Intervention Group Is Significantly Improved Compared with Those in the Control Group}

We also compared QOL of patients in the intervention group and those in the control group. As shown in Table 2 , the scores of the physical function, the psychological function, and the social function for patients in the intervention groups were significantly higher than those in the control group. Consistently, the scores of overall QOL were also higher for patients in the intervention group. In contrast, the scores of common symptoms and side effects were lower for patients in the intervention group (Table 2). Taken together, these results suggest that the psycho-educational intervention improves the psychological, physical, and social function in patients with advanced lung cancer undergoing chemotherapy, and that it also lessen common symptoms and side effects associated with chemotherapy.

\section{Discussion}

To maintain high QOL is an important objective of treatment of patients with advanced cancer.

In this study, we have demonstrated that a purposely designed, nurse delivered psycho-educational intervention significantly improves QOL of patients with advanced lung cancer undergoing chemotherapy. This was not only revealed by the improved physical, psychological, and social function, and overall QOL scores, but was also

Table 1. Comparison of QOL of patients in the intervention group before and after the psycho-educational intervention ${ }^{1}$.

\begin{tabular}{|c|c|c|c|c|c|c|c|c|}
\hline \multirow[t]{2}{*}{ Variables } & \multicolumn{2}{|c|}{$\begin{array}{l}\text { Before the } \\
\text { intervention }\end{array}$} & \multirow[t]{2}{*}{$\bar{X} \pm S$} & \multicolumn{2}{|c|}{$\begin{array}{l}\text { After the } \\
\text { intervention }\end{array}$} & \multirow[t]{2}{*}{$\bar{X} \pm S$} & \multirow[t]{2}{*}{$t$} & \multirow[t]{2}{*}{$\mathrm{p}^{4}$} \\
\hline & $\operatorname{Min}^{2}$ & $\operatorname{Max}^{3}$ & & Min & $\max$ & & & \\
\hline Physical function & 13.00 & 34.00 & $22.03 \pm 3.48$ & 12.00 & 35.00 & $26.01 \pm 3.52$ & 4.347 & 0.026 \\
\hline Psychological function & 21.00 & 55.00 & $37.49 \pm 5.79$ & 19.00 & 50.00 & $30.38 \pm 6.03$ & 10.034 & 0.002 \\
\hline Social function & 15.00 & 28.00 & $21.07 \pm 2.51$ & 14.00 & 30.00 & $22.29 \pm 2.74$ & 1.049 & 0.549 \\
\hline Common symptoms and side effects & 22.00 & 34.00 & $28.77 \pm 2.08$ & 23.00 & 34.00 & $28.06 \pm 2.39$ & 1.982 & 0.380 \\
\hline Specific module & 25.00 & 37.00 & $30.92 \pm 2.35$ & 20.00 & 33.00 & $26.29 \pm 2.61$ & 6.168 & 0.019 \\
\hline Total quality of life scores & 104.00 & 176.00 & $150.48 \pm 15.41$ & 115.00 & 193.00 & $170.23 \pm 15.88$ & 11.493 & $<0.000$ \\
\hline
\end{tabular}

${ }^{1}$ QOL was measured using the one scale method developed by Wan et al. [5];

${ }^{2}$ Minimal values; ${ }^{3}$ Maximal values;

${ }^{4} \chi^{2}$ test was used to compare the difference between groups. A p value $<0.05$ was considered statistically significant. 
Table 2. Comparison of QOL of patients with advanced lung cancer undergoing chemotherapy with or without the psycho-educational intervention ${ }^{1}$.

\begin{tabular}{|c|c|c|c|c|c|c|c|c|}
\hline \multirow{2}{*}{ Variables } & \multicolumn{3}{|c|}{ Intervention group } & \multicolumn{3}{|c|}{ Control group } & \multirow{2}{*}{$t$} & \multirow{2}{*}{$\mathrm{p}^{4}$} \\
\hline & $\operatorname{Min}^{2}$ & $\operatorname{Max}^{3}$ & $\bar{X} \pm S$ & $\min$ & $\max$ & $\bar{X} \pm S$ & & \\
\hline Physical function & 12.00 & 35.00 & $26.01 \pm 3.52$ & 10.00 & 33.00 & $22.47 \pm 2.49$ & 5.948 & $<0.01$ \\
\hline Psychological function & 19.00 & 50.00 & $30.38 \pm 6.03$ & 21.00 & 53.00 & $36.29 \pm 7.19$ & 6.034 & $<0.01$ \\
\hline Social function & 14.00 & 30.00 & $22.29 \pm 2.74$ & 10.00 & 25.00 & $17.87 \pm 2.42$ & 7.049 & $<0.01$ \\
\hline Common symptoms and side effects & 23.00 & 34.00 & $27.06 \pm 2.39$ & 25.00 & 38.00 & $31.88 \pm 2.51$ & 6.367 & $<0.01$ \\
\hline Specific module & 20.00 & 33.00 & $26.29 \pm 2.61$ & 22.00 & 38.00 & $30.43 \pm 3.09$ & 8.741 & $<0.01$ \\
\hline The total quality of life scores & 115.00 & 193.00 & $170.23 \pm 15.88$ & 103.00 & 168.00 & $142.47 \pm 13.01$ & 23.49 & $<0.01$ \\
\hline
\end{tabular}

${ }^{1}$ QOL was measured using the one scale method developed by Wan et al. [5];

${ }^{2}$ Minimal values; ${ }^{3}$ Maximal values;

${ }^{4} \chi^{2}$ test was used to compare the difference between groups. A p value $<0.05$ was considered statistically significant.

shown by reduced common symptoms and adverse effects associated with chemotherapy. Noticeably, scores for cough also appeared lower in patients after the psycho-educational intervention, but whether this was due to implementation of the intervention or other factors remains to be further clarified. Regardless, Results from this study suggest that nurses can play an important role in improving QOL of patients with advanced lung cancer who undergo chemotherapy by providing systemic psycho-educational interventions.

Although psychological interventions to improve QOL of lung cancer patients has received increasing attention in recent years, there have been only limited numbers of reports in the literature about QOL of lung cancer patients in China, where lung cancer is becoming the leading cause of cancer-related death [5]-[7]. This is conceivably related to the large population and relatively few psychologists specialized in the field of QOL of cancer patients in China. The results from this study showing that a psycho-educational intervention can be delivered by trained nurses to late-stage lung cancer patients undergoing chemotherapy have important practical implications, in that this is a highly feasible and cost-effective way that can be carried out in large scales independently of the availability of specialized psychologists. In addition, this approach can be readily adapted to the management of patients with other types of cancers who undergo systemic treatment.

\section{Conclusion}

Psycho-educational interventions delivered by nurses are a useful strategy to improve QOL of late-stage lung cancer patients undergoing chemotherapy, which are also conceivably applicable to patients with other types of advanced cancers.

\section{Conflicts of Interest}

The authors declare no conflict of interest.

\section{References}

[1] Chen, W., Zheng, R., Zeng, H. and Zhang, S. (2015) Epidemiology of Lung Cancer in China. Thoracic Cancer, 6, 209215. http://dx.doi.org/10.1111/1759-7714.12169

[2] Lemjabbar-Alaoui, H., Hassan, O., Yang, Y.W. and Buchanan, P. (2015) Lung Cancer: Biology and Treatment Options. Biochimica et Biophysica Acta, 1856, 189-210. http://dx.doi.org/10.1016/j.bbcan.2015.08.002

[3] Gerber, D.E. and Schiller, J.H. (2013) Maintenance Chemotherapy for Advanced Non-Small-Cell Lung Cancer: New Life for an Old Idea. Journal of Clinical Oncology, 31, 1009-1020. http://dx.doi.org/10.1200/JCO.2012.43.7459

[4] Wang, D. and Fu, J. (2014) Symptom Clusters and Quality of Life in China Patients with Lung Cancer Undergoing Chemotherapy. African Health Sciences, 14, 49-55. http://dx.doi.org/10.4314/ahs.v14i1.8

[5] Wan, C., Zhang, C., Li, G., Shen, L., Luo, J., Yang, Z. and Meng, Q. (2007) Development and Psychometric Properties of the System of Quality of Life Instruments for Cancer Patients: Lung Cancer QLICP-LU. Bulletin of Chinese Cancer, 11, 858-861

[6] Geater, S.L., Xu, C.R., Zhou, C., Hu, C.P., Feng, J., Lu, S., Huang, Y., Li, W., Hou, M., Shi, J.H., Lee, K.Y., Palmer, 
M., Shi, Y., Lungershausen, J. and Wu, Y.L. (2015) Symptom and Quality of Life Improvement in LUX-Lung 6: An Open-Label Phase III Study of Afatinib Versus Cisplatin/Gemcitabine in Asian Patients With EGFR Mutation-Positive Advanced Non-small-cell Lung Cancer. Journal of Thoracic Oncology, 10, 883-889.

http://dx.doi.org/10.1097/JTO.0000000000000517

[7] Ma, Y., Yang, Y., Huang, Y., Zhao, H., Hou, X., Tian, Y., Zhao, Y., Xue, C., Fang, W., Wu, X., Hu, Z., Liang, W., Zhou, T., Qin, T. and Zhang, L. (2014) An Investigation of Symptom Burden and Quality of Life in Chinese ChemoNaïve Advanced Lung Cancer Patients by Using the Instrument-Cloud QOL System. Lung Cancer, 84, 301-306.

http://dx.doi.org/10.1016/j.lungcan.2014.01.027 